\title{
Three differently-colored polymorphs of a diketopyrrolopyrrole derivative having butyl groups
}

\author{
T. Muroya ${ }^{1}$, N. Okada ${ }^{1}$, A. Toda ${ }^{2}$, K. Imai ${ }^{2}$, T. Sekine ${ }^{2}$, S. Matsumoto ${ }^{1}$

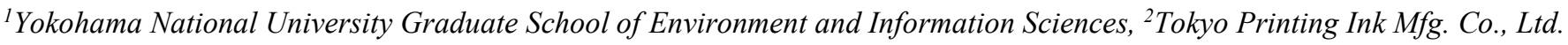 \\ matsumoto-shinya-py@ynu.ac.jp
}

Diketopyrrolopyrrole (DPP) is an industrially important dye. It is also expected to be used as a functional dye, and a lot of research has been done on its various applicability. The introduction of flexible alkyl groups into the amino groups of DPP is reported to lead polymorph occurrence. [1][2] A derivative of DPP in which both amino groups are substituted with a butyl group are found to exhibit three differently coloured polymorphs (red, orange, and yellow). All polymorphs could be obtained individually using the liquid-liquid diffusion method with chloroform as the good solvent and $n$-hexane as the poor solvent. The preparation of the red and yellow forms was easy, whereas the orange polymorphs was infrequently obtained. The results of the crystal structure analysis indicate that the asymmetric unit of the red and yellow polymorphs is one molecule, and that of the orange polymorph is two molecules. The molecular conformation of the three polymorphs is shown in Fig. 1. The red and orange polymorphs have butyl groups extending to both sides of the molecular plane. The two butyl groups of the yellow polymorph were found to project out from the molecular plane in the same direction. The crystal structures of the three polymorphs are shown in Fig. 2. The molecules in the red polymorph are stacked along the $a$-axis. The orange crystal has two asymmetric units (illustrated as green and yellow molecules in Fig. 2), and each asymmetric unit is stacked along the $b$-axis. The yellow crystals formed a chain-like structure with the $\pi$-conjugated planes facing each other. The detailed comparison of their crystal structures and their optical and thermal properties will be presented.

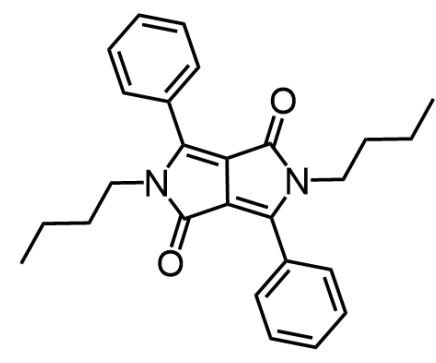

(a)

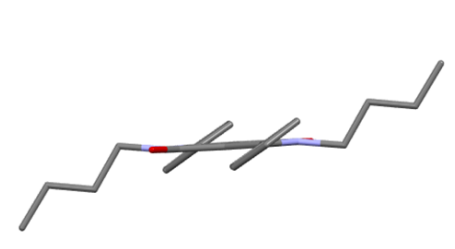

(b)

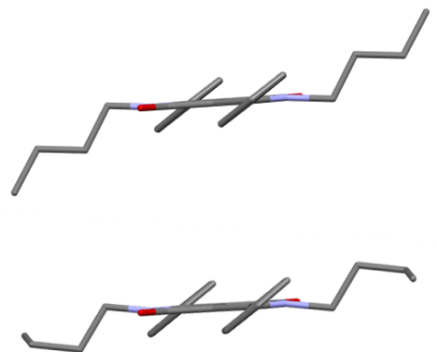

(c)

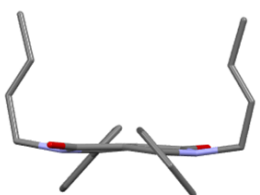

(d)

Figure 1. (a) Molecular structure of the DPP derivative and molecular geometries of (b) red, (c) orange (having two asymmetric units), and (d) yellow polymorphs viewing along the central $\mathrm{C}-\mathrm{C}$ bond of the DPP skeleton.

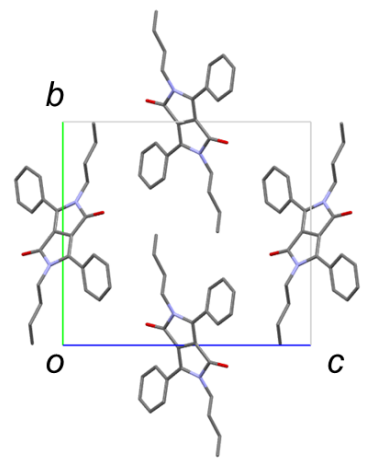

(a)

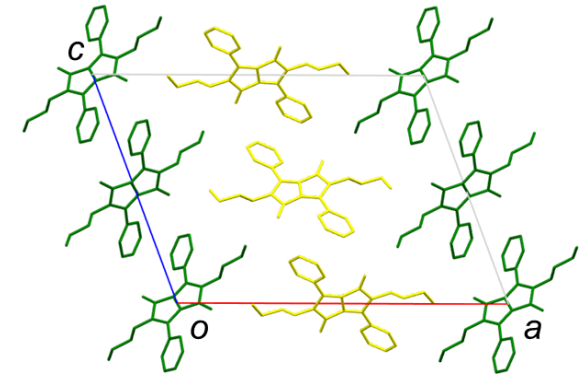

(b)

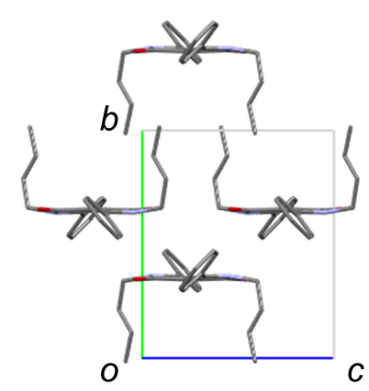

(c)

Figure 2. Molecular arrangement of the three polymorphs, (a) red, (b) orange, (c) yellow.

[1] So H. \& Matsumoto S., (2019). Acta Cryst. B75, 414.

[2] Kikuchi Y. \& Matsumoto S., Cryst. Eng. Comm. in press.

Keywords: Diketopyrrolopyrrole; Polymorph; X-ray Structural Analysis; Optical Property; Thermal Property Acta Cryst. (2021), A77, C1032 